\title{
Modelling of the Current-Voltage Characteristics of the HTc Superconducting Tapes Subjected to the Bending Strain Process
}

\author{
J. SOSNOWSKI* \\ Electrotechnical Institute, M. Pożaryskiego 28, 04-703 Warsaw, Poland \\ (Received July 4, 2018; in final form November 28, 2018)
}

\begin{abstract}
In the paper the current-voltage characteristics of the HTc superconducting tapes subjected to the bending strain process have been analyzed. The variation of the strain acting on the superconducting element, a filament in the first generation $(1 \mathrm{G})$ or a film in the second generation $(2 \mathrm{G})$ composite tape, in respect of the applied bending strain, has been considered. The elasticity properties of tape and geometry of micro-cracks have been taken then into account. Decreasing with length along the tape solution of the equations describing current and voltage distribution in composite bent superconducting tape, with micro-cracks has been found and used for determining current-voltage characteristics. For description of these characteristics theoretical model in the case of the critical current determined by the flux creep effect and overall capturing of the vortices on the structural defects has been proposed. The model has been fitted to the experimental current-voltage characteristics of a Bi-based tape subjected to the bending strain. Fairly good agreement between experiment and theoretical calculations has been obtained.
\end{abstract}

DOI: 10.12693/APhysPolA.134.1194

PACS/topics: current-voltage characteristics, bending strain, HTc superconducting tapes

\section{Introduction}

In numerous applications superconducting tapes are subjected to the bending strain, in which they are mechanically deformed. It relates especially to the process of manufacturing of HTc superconducting coils. Applying winding technology leads to the creation of the microcracks, whose shape will differ from that of micro-cracks arising usually due to mechanical elongation process, simply approximated by regular cuboid. Arising in the bending strain process micro-cracks, created in the superconducting elements of HTc tapes, filaments in the first generation $(1 \mathrm{G})$ or films in the second generation $(2 \mathrm{G})$ composite tape, should have rather form of a bent cuboid with tilted external walls. It will be shown in the paper in which way the bending strain influences the currentvoltage characteristics of HTc superconducting tapes taking into account this geometrical effect. This issue will be considered in the present paper in the model of the entirely captured pancake vortices on nanosized defects and for new solution describing current and voltage distribution in the bent superconducting tape.

\section{Analysis of the bending strain influence on the current-voltage characteristics}

The bending strain effect appearing especially in the technological works performed during the preparation of the superconducting coils has great technical as well as

*corresponding author; e-mail: j.sosnowski@iel.waw.pl scientific importance. On the significance of this effect there indicates Fig. 1, in which are shown results of our measurements performed on the BiSCCO tape, of the influence bending strain on the critical current density of the HTc superconducting tape. The measurements were performed on the multifilamentary tape of the first generation prepared by the powder in tube method of the approximated dimensions $0.3 \times 4 \mathrm{~mm}$. The tape consisted from Bi-2223 oxide filaments immersed in silver sheath. Tape bending process was applied in room temperature on especial round forms with various radii. Current-voltage characteristics have been measured and critical current density for each bending strain value determined. In Fig. 1 there are shown the critical current densities determined in this way, reduced to the critical current density without bending, as the function of the applied bending strain. Such presentation of the critical current densities in reduced units allows to estimate directly in percentages the size of the changes caused by the bending strain process. Dots denote the experimental results, while solid lines describe theoretical approximations, discussed below. In the present paper a new approach to the analysis of the influence of the bending strain on the current-voltage characteristics of the superconducting tapes is given, which takes into account influence of the geometrical factor on bending strain acting on filaments. The issue of the influence of the elongation induced strain on the current-voltage characteristics has been investigated in [1]. Others experimental works devoted to the subject of strain in superconductors mainly are given in [2-10] and indicate the importance of discussed here problems. Observed in the present paper strong dependence of the critical current on the bending 


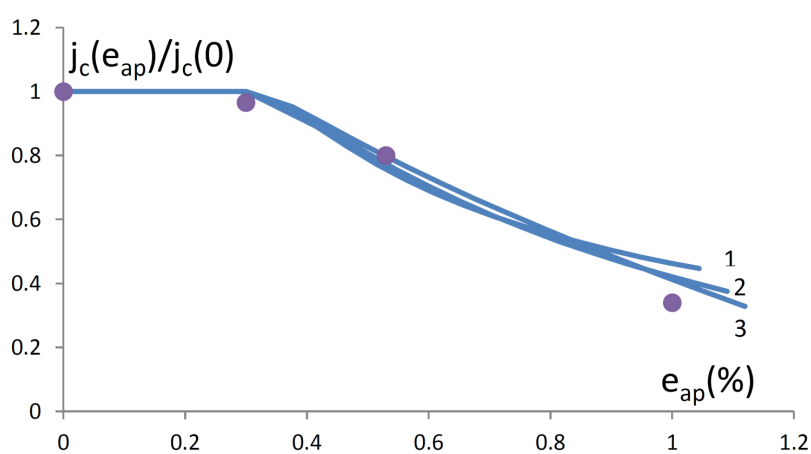

Fig. 1. Influence of the bending strain on the reduced critical current density, for $1 \mathrm{G}$ Bi-based superconducting tape, measured in the liquid nitrogen temperature. Solid lines denote the analytical approximations of the experimental results: 1 - fractional, 2 - exponential, and 3 - linear, according to the relations (1)-(3).

strain for a Bi-based tape shown in Fig. 1 is in qualitative agreement with the experimental data given recently in [2].

Dependence of the reduced critical current density versus the bending strain $e_{a p}$, shown in Fig. 1 describes the rupture probability function $G\left(e_{a p}\right)$, defined as $G\left(e_{a p}\right)=1-j_{C}\left(e_{a p}\right) / j_{C}(0)$, which has been fitted to experimental results through: (1) fractional, (2) exponential, and (3) linear approximations, according to the formulae

$$
\begin{aligned}
& G\left(e_{a p}\right)=1-\left[1.036+1.72\left(e_{a p}-e_{c}\right)\right]^{-1}, \\
& G\left(e_{a p}\right)=1-\exp \left(1.4\left(e_{c}-e_{a p}\right)\right), \\
& G\left(e_{a p}\right)=0.96\left(e_{a p}-e_{c}\right) .
\end{aligned}
$$

Bending strain applied to the total tape $e_{a p}$ given in percent, is defined according to the following formula:

$$
e_{a p}(\%)=100 \frac{t}{D},
$$

where $t$ is thickness of the superconducting tape, while $D$ is diameter of the bending, $e_{c}=0.25 \%$ is the critical value of the bending strain, for which superconducting tape still retains fully its elastic properties. Equations (1)-(3) are valid therefore for $e_{a p}>e_{c}$,

As follows from Fig. 1, for bending strain smaller than critical one $e_{c}$, in superconducting tape appears an elastic, reversible region, in which micro-cracks do not arise, therefore critical current density is constant and as shown in Fig. 2 rupture probability function disappears $G\left(e_{a p}<e_{c}\right)=0$. For larger bending strain the microcracks in the tape arise, due to the breaking forces acting on it. The strain affects then immersed in the silver matrix superconducting filaments or films and rupture probability function $G\left(e_{a p}\right)$ increases, as presents Fig. 2 for linear, exponential and fractional approximations. Creation of the micro-cracks constricts the transport of the electric current through the superconductor and dimin-

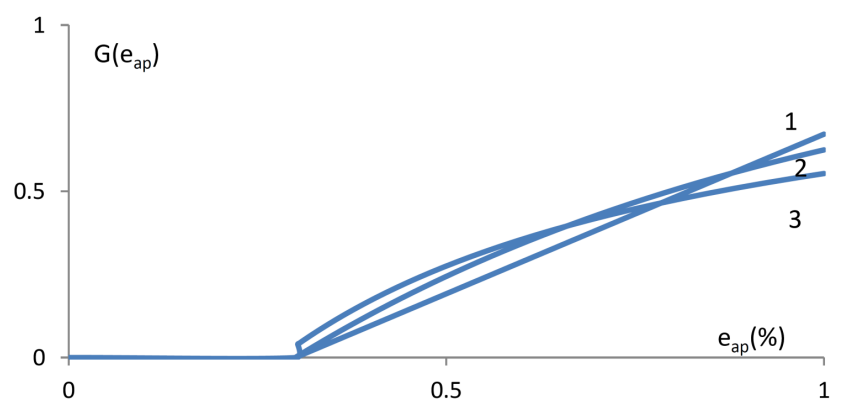

Fig. 2. Rupture probability function $\mathrm{G}\left(\mathrm{e}_{a p}\right)$ as a function of the applied bending strain $\mathrm{e}_{a p}$ for: 1 - linear, 2 - exponential, and 3 - fractional approximation.

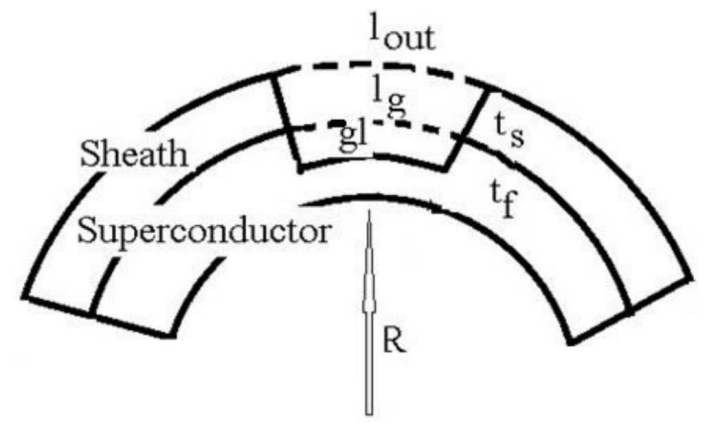

Fig. 3. Schematic view of the formation of the microcracks as the result of the bending strain procedure applied to HTc superconducting tape. Descriptions of the symbols used here are given in the text.

ishes the critical current density, which is shown by experimental results given in Fig. 1. The elasticity properties of the silver sheath and geometrical factor modify the strain acting on the superconducting elements film or filaments, in comparison to the total applied to the composite superconducting tape bending strain and therefore the length of the micro-cracks. In the model it has been assumed that the length of the micro-cracks per unit length of a tape is proportional to the difference between applied to the tape bending strain $e_{a p}$ and total strain acting on the superconducting element $e_{\text {suf }}$. In the approximation presented in Fig. 3, thickness of tape is equal $t=t_{f}+t_{s}$, where $t_{f}$ is thickness of superconducting element (film or filaments), $t_{s}$ thickness of silver sheath. Other symbols used in Fig. 3 are: $l_{\text {out }}$ the length of the micro-crack on the surface of superconducting tape, $l_{g}$ this length at the surface of superconducting element, which as follows from Fig. 3 are not the same values, $g l$ is the depth of the micro-crack in the superconducting element. $R$ denotes bending radius, related to the average bending diameter of the tape $D$ and superconducting element: thin film or filament, bending diameter $D_{f}$ through the relation

$$
\begin{aligned}
& D=2 R+t_{f}+t_{s}, \\
& D_{f}=2 R+t_{f} .
\end{aligned}
$$


According to Fig. 3, it is considered here a superconducting tape composed of thin superconducting film covered by the normal metal - silver sheath, which is the case of the second generation $(2 \mathrm{G})$ superconducting tape. This picture can be applied also to modelling of the electromagnetic properties of the first generation tapes, if treating these parameters as average values describing the filling of the tape by a superconductor and normal matrix volume. As it is shown in Fig. 3 the length of the micro-cracks, marked by broken line, arising due to a bending strain, is different on the surface of the tape and inside the superconductor. According to the geometry the bending strain, acting on the superconducting element $e_{\text {supg }}$ is defined as:

$$
e_{\text {supg }}=100 \frac{t_{f}}{D_{f}}=100 \frac{t_{f}}{D-t_{s}} .
$$

As follows therefore from Eqs. (4)-(7) the strain $e_{\text {supg }}$ acting on the superconducting element is modified due to pure geometrical reasons in respect to the applied to the total tape bending strain $e_{a p}$ given by Eq. (4), which is described by the next relation

$$
e_{\text {supg }}=\frac{e_{a p}}{1+q\left(1-\frac{e_{a p}}{100}\right)} .
$$

Symbol $q$ appearing in Eq. (8) is defined according to:

$$
q=\frac{t_{s}}{t_{f}}
$$

and describes the geometrical ratio between effective thicknesses of superconducting and stabilizing elements in HTc tape, it is fulfilment of the tape by superconductor.

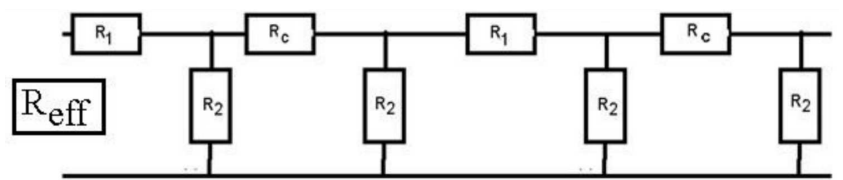

Fig. 4. Scheme of the deformed multi-composite superconducting tape of effective resistance per unit length $R_{\text {eff }}$ in the form of the long line of resistors joined in a row $\mathrm{R}_{1}$ - describing the undeformed regions and $R_{c}$ regions with micro-cracks and parallel through the silver sheath of resistance $R_{2}$.

The regular ordering of the micro-cracks in a tape allows to treat the tape as a long line of deformed elements interlaced with the second long line containing undeformed ones. The tape with the micro-cracks can be approximated [1] by a long line of electrical resistors, corresponding to the superconductors in the resistive state, as is shown in Fig. 4. There are presented here two coupled long lines of resistors of different resistance per unit length: $R_{c}$ with smaller cross-section due to existence of micro-cracks and $R_{1}$, which corresponds to the resistance, per unit length, of the non-deformed regions. Both these lines are joined to the ground through the silver matrix of resistance $R_{2}$.

It is easy to split the resistors arrangement as shown in Fig. 4 into two individual long lines of the undeformed and deformed resistors. It then allows to find the simple mathematical relations describing variation of the voltage and current along such long lines, while taking into account the leakage of the current through normal matrix and voltage drop with the distance inside the line. The relations $\mathrm{d} V / \mathrm{d} x=-R_{1} I$ and $\mathrm{d} I / \mathrm{d} x=-V / R_{2}$ in undeformed region lead to the dependences

$$
\frac{\mathrm{d}^{2} I}{\mathrm{~d} x^{2}}=\frac{R_{1}}{R_{2}} I, \quad \frac{\mathrm{d}^{2} V}{\mathrm{~d} x^{2}}=\frac{R_{1}}{R_{2}} V .
$$

The solution of Eqs. (10) describing the decrease of current with distance along the tape, connected with the leakage of the current to the sheath has been found in the exponential form as

$$
I(x)=I(0) \exp \left(-\sqrt{\frac{R_{1}}{R_{2}}} x\right) .
$$

Similar solution describes the voltage variation along the tape

$$
V(x)=I(0) \sqrt{R_{1} R_{2}} \exp \left(-\sqrt{\frac{R_{1}}{R_{2}}} x\right) .
$$

Effective resistance per unit length of this long line is given by

$$
R_{\text {effu }}=\sqrt{R_{1} R_{2}} .
$$

In the deformed, by the existence of the micro-cracks, long line the appropriate relations describing current $\mathrm{d} I / \mathrm{d} x=-V / R_{2}$ and voltage $\mathrm{d} V / \mathrm{d} x=-R_{c} I$ distributions with the distance along the tape lead to the analogous relations

$$
\frac{\partial^{2} I}{\partial x^{2}}=\frac{R_{c}}{R_{2}} I .
$$

Solution of Eq. (14) has been analogously to Eqs. (10) proposed in the exponential form

$$
I(x)=I(0) \exp \left(-\sqrt{\frac{R_{c}}{R_{2}}} x\right)
$$

and the voltage variation has been determined by

$$
V(x)=I(0) \sqrt{R_{c} R_{2}} \exp \left(-\sqrt{\frac{R_{c}}{R_{2}}} x\right),
$$

while effective resistance per unit length of this deformed part of the tape is:

$$
R_{\text {effd }}=\sqrt{R_{c} R_{2}} \text {. }
$$

The final expression for the effective resistance, per unit length of the tape in resistive state, containing undeformed and deformed regions is

$$
R_{\mathrm{eff}}=\sqrt{R_{1}\left(1-l_{e}\right) R_{2}}+\sqrt{R_{c} l_{e} R_{2}},
$$

where $l_{e}$ is the effective length of deformed regions in the unit length of the tape. As follows from Eq. (18) in order to find the effective resistance of the deformed tape, $R_{\text {eff }}$, it is necessary to know nonlinear resistances of the tape $R_{1}$ and $R_{c}$ and also effective length of the deformed regions $l_{e}$. Resistance of the silver sheath $R_{2}$ is treated as constant. Resistances $R_{1}$ and $R_{c}$ connected with the undeformed section of the tape and the deformed one, respectively, are determined [1] through the relations 


$$
R_{1}=E(I / S) / I, R_{c}=E\left(\frac{I}{S\left(1-G\left(e_{a p}\right)\right)}\right) / I,
$$

in which $E(j)$ is just the current-voltage characteristic of the unit length of the superconducting tape, of the crosssection $S$, while $j$ is electric current density $j=I / S$, where $I$ is the transport current. Right-hand side part of Eq. (19) gives the current-voltage characteristic $E(j)$ divided on current $I$ for tape with micro-cracks, so with smaller cross-section, which is now described by the product $S\left(1-G\left(e_{a p}\right)\right)$. Here $G\left(e_{a p}\right)$ is rupture probability function given previously by Eqs. (1)-(3).

In the present paper the case of layered HTc superconductors has been investigated, in which appear the pancake vortices. These vortices can be generated by external magnetic field or transport current flow. The currentvoltage characteristics have been determined basing on the analysis of the pinning interaction of the pancake vortices with the structural defects, as it shows Fig. 5. This picture can be extended also on Josephson-like vortices, taking into account the interlayers interactions but it is beside the scope of the present paper.

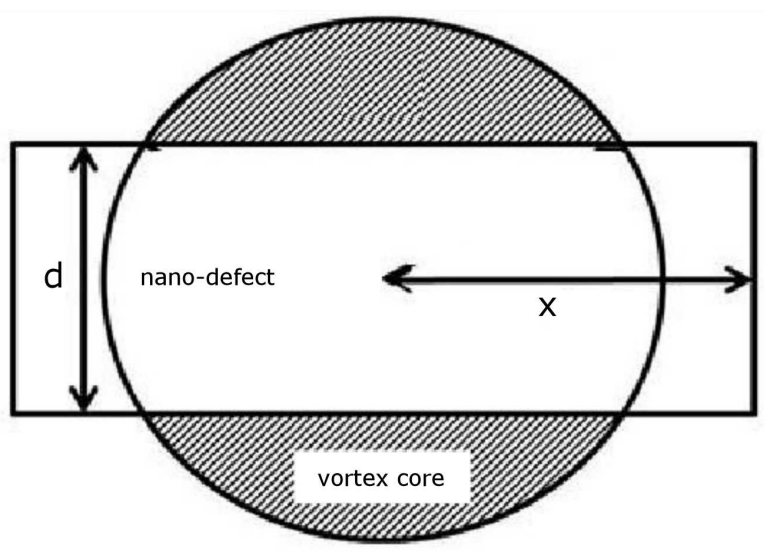

Fig. 5. View of the captured core of the pancake type vortex on the nanosized defects. Shadowed region indicates the vortex core, while rectangle the cross-section of the capturing nanosized center.

In Fig. 5 there is shown the pancake vortex core of the size $2 \xi$, where $\xi$ is the coherence length, captured on the nanosized defect. The surrounding this core superconducting currents are neglected in this figure, because in this model more widely presented in [11], main contribution to the system energy of captured vortex arises from the vortex core, being in the normal state. Geometry presented in Fig. 5 is especially favorable for thin nanodefects of width $d$ smaller than $2 \xi$, because superconductivity will be then continuously induced inside of the nanodefect by the proximity effect. Such approximation is especially useful for irradiation induced nanodefects. For considered here case of the total capturing of the vortices the appropriate pinning potential barrier $\Delta U$ after applying the renormalization procedure [11] is given by

$$
\begin{aligned}
& \Delta U(i)=\frac{\mu_{0} H_{c}^{2}}{2} l_{a} \xi^{2} \\
& \times\left\{-\arcsin (i)+2 \arcsin \left(\frac{d}{2 \xi}\right)+\frac{d}{\xi} \sqrt{1-\left(\frac{d}{2 \xi}\right)^{2}}\right. \\
& \left.+i\left[\frac{\pi}{2}-2 \arcsin \left(\frac{d}{2 \xi}\right)-\frac{d}{\xi} \sqrt{1-\left(\frac{d}{2 \xi}\right)^{2}}-\sqrt{1-i^{2}}\right]\right\} .
\end{aligned}
$$

Equations (20) describes the pinning potential barrier [11], which holds the captured pancake vortex against the Lorentz force breaking up vortices in the flux creep process. Here $i=j / j_{c}$ is transport electric current density reduced to the critical current density $j_{c}$. For $i=1$ potential barrier disappears and vortices start move in the flux flow motion. $l_{a}$ is thickness of the capturing center, which for the case of the layered superconductor, corresponds to the thickness of the superconducting layer, while $H_{c}$ is the thermodynamic critical magnetic field. Expression (20) for the pinning potential barrier $\Delta U$ is inserted then into basic equation describing the currentvoltage characteristics of the HTc superconductor for flux creep forward and backward processes, according to relation

$$
E=-B \omega p\left(\mathrm{e}^{\frac{-\Delta U(0)(1+i)}{k_{\mathrm{B}} T}}-\mathrm{e}^{\frac{-\Delta U(i)}{k_{\mathrm{B}} T}} .\right)
$$

In Eq. (21) $E$ is electric field, $T$ is temperature, $k_{\mathrm{B}}$ Boltzmann's constant, while $p$ averaged distance between pinning centers, in the present case structural defects [11], $\omega$ is hopping frequency, it is number of jumps in time unit of the pancake vortices between the nearest pinning centers. For determining the current-voltage characteristics of the tape with the strain introduced in the bending process it is necessary to estimate the effective length of the deformed regions. For this purpose the elasticity properties of the superconducting composite tape have been considered, which are modified in the bending deformation procedure. It is shown in Fig. 6 which presents the scheme of the tape from the point of view of its elastic properties. While bending strain $e_{a p}$ is applied to the tape, on the superconducting elements is acting lower strain $e_{\text {sup }}$ due to the elastic properties of silver matrix $e_{\text {sheath}}$, as it will be shown in Figs. 7-9.

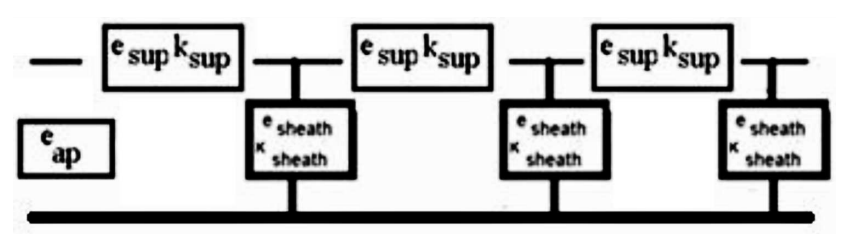

Fig. 6. The distribution of the elasticity parameters in the bent superconducting composite tape.

Similar situation concerns the spring elasticity constant $k$ of the tape, so in fact it appears here the analogy to the Fig. 4 presenting the resistive model of the composite superconducting tape [1]. In this analogy 
total bending strain $e_{a p}$ corresponds to the effective resistance $R_{\text {eff }}$, while strain acting on the superconducting element of the tape (film or filaments) denoted as $e_{\text {sup }}$ corresponds to the resistance $R_{1}$. Similar are relations describing the spring constants of superconducting element and total tape, which are joined through

$$
\frac{e_{\text {sup }}}{e_{a p}}=\frac{R_{\text {eff }}}{R_{1}}, \quad \frac{k_{\text {sup }}}{k_{\text {sheath }}}=\frac{R_{1}}{R_{2}} \text {. }
$$

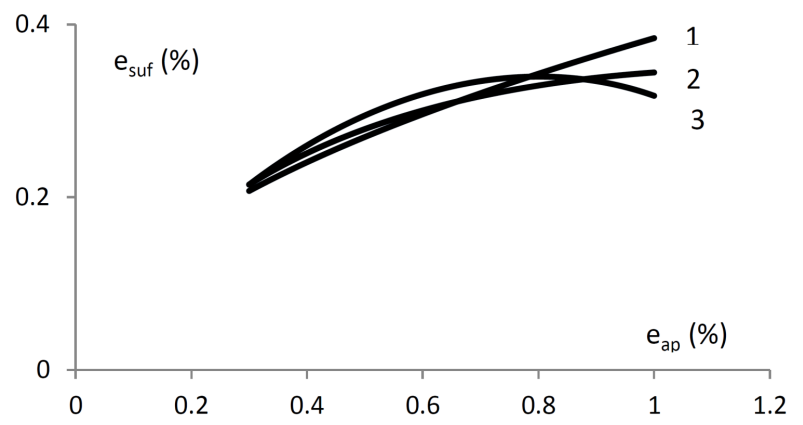

Fig. 7. Comparison of the dependence of the total strain acting on superconducting element versus applied to the tape bending strain for different rupture probability functions: 1 - fractional, 2 - exponential, and 3 - linear approximation.

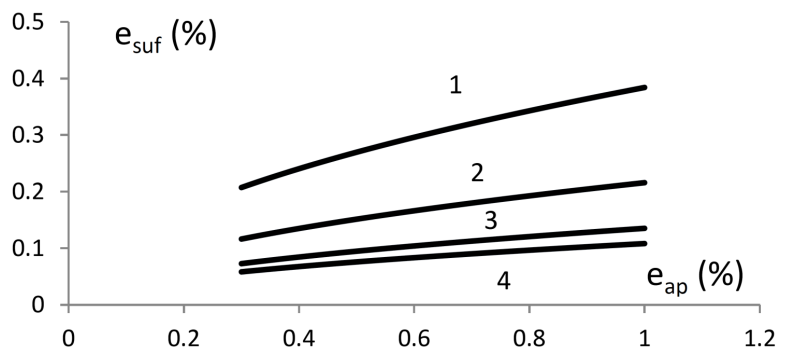

Fig. 8. The dependence of the total strain $e_{\text {suf }}$ acting on a superconducting element as a function of the applied to the tape bending strain $e_{a p}$ for parameters $q$ : 1 $-q=0.4,2-q=1.5,3-q=3,4-q=4$ and for fractional approximation of the rupture probability function $G\left(e_{a p}\right)$.

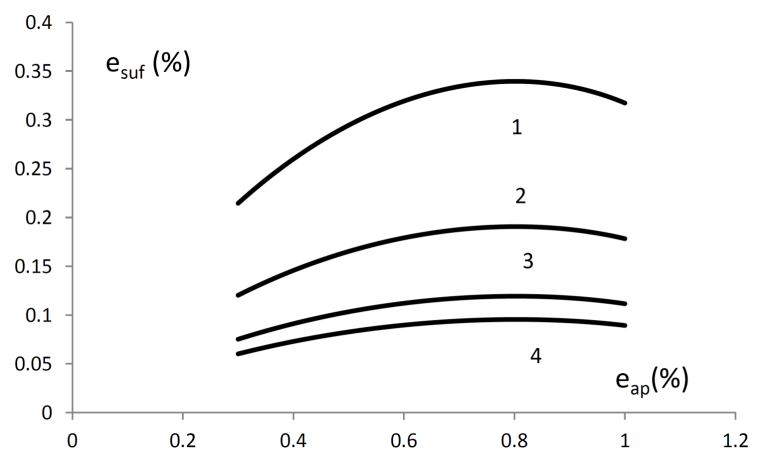

Fig. 9. As in Fig. 8, but for linear approximation of the rupture probability function.
From the mutual dependences (22) it follows that there is an analogy between parameters $k$ and $R$. It appears however inverse proportionality between strain $e$ and spring elasticity constant $k$, which follows from the fact that while stress is applied then arise micro-cracks in superconducting elements and elasticity forces are sustained by the silver matrix. Its elastic properties are therefore suppressed with enhancement of rupture probability function $G(e)$. This dependence has been described here by the following formula:

$$
k_{\text {sheath }} \approx k_{\text {sup }} \frac{1-G\left(e_{a p}\right)}{1+G\left(e_{a p}\right)} .
$$

From this dependence and relations (22) we obtain the expression for the strain acting on the superconducting element in the tape $e_{\text {sup }}$, the part connected with elasticity properties of the multi-composite tape, as the function of the applied strain $e_{\mathrm{ap}}$ :

$$
e_{\mathrm{sup}} \approx e_{a p} \sqrt{\frac{1-G\left(e_{a p}\right)}{1+G\left(e_{a p}\right)}} .
$$

Total strain $e_{\text {suf }}$ acting on superconducting element of the tape will be obtained, if to consider also the geometrical factor given by Eq. (8). The result brings the form

$$
e_{\mathrm{suf}} \approx \frac{e_{a p}}{1+q\left(1-\frac{e_{a p}}{100}\right)} \sqrt{\frac{1-G\left(e_{a p}\right)}{1+G\left(e_{a p}\right)}} .
$$

Dependences describing the total strain acting on a superconducting element $e_{\text {suf }}$ as a function of the applied bending strain $e_{\text {app }}$ for various shapes of rupture probability functions and material parameter $q$, defined in Eq. (9), of a composite tape are shown in Figs. 7-9.

Performed analysis allows already for evaluation of the relative length of the micro-cracks, per unit length of a tape, which value was assumed to be proportional to the difference between the applied bending strain to the tape $e_{a p}$ and strain acting on a superconducting element $e_{\text {suf }}$. The relative length of deformed regions $l_{e}$, on the halfdepth of micro-cracks in superconducting element, in the unit length of the deformed tape is

$$
\begin{aligned}
l_{e} & =\frac{2 a-1-G\left(e_{a p}\right) \frac{t_{f}}{t_{s}}}{2 a+1} c e_{a p} \\
& \times\left(1-\sqrt{\frac{1-G\left(e_{a p}\right)}{1+G\left(e_{a p}\right)}} \frac{1}{1+q\left(1-\frac{e_{a p}}{100}\right)}\right),
\end{aligned}
$$

where $c$ is a proportionality coefficient, while parameter $a$ is defined as:

$$
a=\frac{50}{e_{a p}}\left(\frac{t_{f}}{t_{s}}+1\right)+\frac{t_{f}}{2 t_{s}} .
$$

Because as it follows from Fig. 3 created in the bending strain process micro-cracks have the form of bent cuboid the length of micro-crack inside the superconducting element, which in fact controls the current in the tape, will slightly distinguish from the length of the microcracks $l_{\text {out }}$ on the surface of tape. The scale of this effect describes parameter $\Delta I_{a v}=l_{e} / l_{\text {out }}$, whose changes are shown graphically in Fig. 10. 


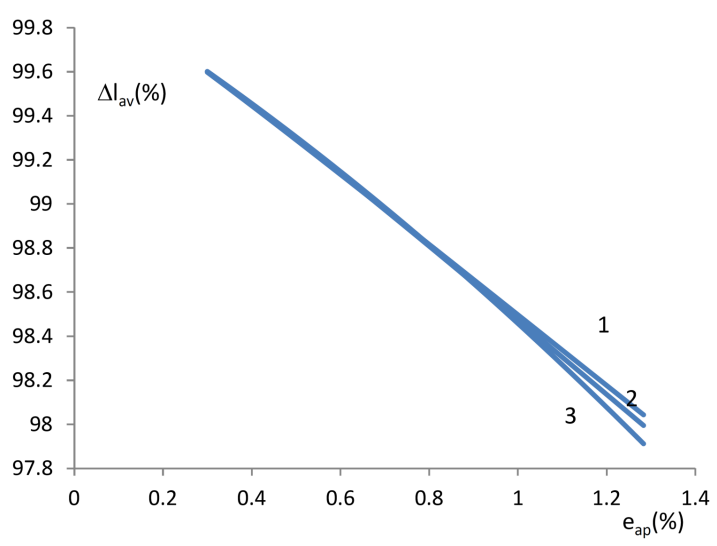

Fig. 10. Ratio of the average length of the micro-cracks in the superconducting element to the length of the micro-cracks on the surface of tape $\Delta I_{a v}$ versus the bending strain applied to the tape for: 1 - fractional, 2 - exponential, and 3 - linear approximation of the rupture probability function.

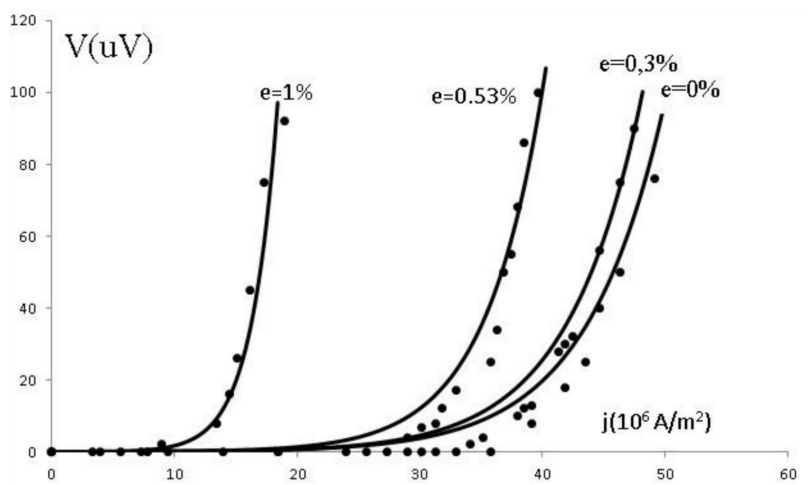

Fig. 11. The comparison of the experimental currentvoltage characteristics of the bent HTc superconducting first generation tape (dots) and theoretically calculated curves (solid lines).

Above considerations allow then to determine the current-voltage characteristics of a bent superconducting tape and compare obtained theoretical results with the experimental data. Measurements of the current-voltage characteristics of bent superconducting Bi-2223 multifilamentary tape shown in Fig. 11 were performed in liquid nitrogen cryostat. Four electric contacts method was used, in which direct current flowed through the tape and voltage has been then detected using sensitive micro-voltmeter. The bending procedure was applied in room temperature, with the help of the especial mechanical, plastic forms of various diameters, to which sample tape was mounted. The total setup was immersed then into liquid nitrogen. The results of fitting theoretical calculations to experimental characteristics measured for various bending strains are shown in Fig. 11, which indicates that the shape of the theoretical current-voltage characteristics is in agreement with experiment and confirms presented modelling procedure. Used in the calculations values of bending strains $e_{a p}$ equal respectively to $0,0.31$, 0.48 and $1.4 \%$ and are also in quite good correlation with the experimentally applied bending strains indicated in this figure.

\section{Conclusions}

Performed theoretical analysis of the current-voltage characteristics of the bent HTc superconducting tape brought the results well corresponding to experimental data measured at liquid nitrogen temperature. Elaborated model allows to predict the variation of critical current of composite superconducting tape with bending strain, which result has also technical importance [12]. Model indicates on the influence on the current-voltage characteristics of bent superconducting tape, beside usual physical parameters as temperature and magnetic field, also such material parameters as filling it with superconducting elements and shape of the rupture probability function.

\section{References}

[1] T. Kiss, H. van Eck, B. ten Haken, H.H.J. ten Kate, IEEE Trans. Appl. Supercond. 11, 3888 (2001).

[2] A. Panchal, A. Bano, M. Ghate, P. Raj, S. Pradhan, J. Phys. Conf. Ser. 823, 012045 (2017).

[3] P. Kovác, M. Dhallé, T. Melišek, H.J.N. van Eck, W.A.J. Wesse, B. ten Haken, I. Hušek, Supercond. Sci. Technol. 16, 600 (2003).

[4] H.-S. Shin, M.J. Dedicatoria, J.R. Dizon, H.S. Ha, S.-S. Oh, Physica C Supercond. 469, 1467 (2009).

[5] M.A. Morsy, K. Sekkina, K.M. Elsabawy, Mater. Sci. Eng. B 103, 71 (2003).

[6] A. Nijhuis, Y. Ilyin, S. Wessel, E. Krooshoop, L. Feng, Y. Miyoshi, IEEE Trans. Appl. Supercond. 19, 1516 (2009).

[7] S. Awaji, K. Watanabe, K. Katagiri, Supercond. Sci. Technol. 16, 661 (2003).

[8] P. Kováč, I. Hušek, T. Melišek, M. Kulich, L. Kopera, Supercond. Sci. Technol. 29, 045002 (2016).

[9] H.S. Shin, K. Katagiri, Supercond. Sci. Technol. 16, 1012 (2003)

[10] T. Takao, T. Iwamura, Y. Fukasawa, S. Minowa, H. Sato, T. Asano, A. Ishiyama, J. Kato, T. Machi, K. Nakao, Y. Shiohara, IEEE Trans. Appl. Supercond. 17, 3513 (2007).

[11] J. Sosnowski, Mod. Phys. Lett. B 30, 1650387 (2016).

[12] J. Sosnowski, Superconducting Cryocables, Book Publisher of Electrotechnical Institute, 2012, p. 1 (in Polish). 